Objectives The objective was to assess the distribution and possible associations of HLA-DRB1 alleles in the Russian population with long standing rheumatoid arthritis.

Methods Forty four patients (pts) with RA ( 2 males, 42 females, mean age $53.3 \pm 10.85 \mathrm{yr}$. and mean disease duration $12.9 \pm 7.71$ yr. and 135 controls were typed for HLA-DRB1 alleles using PCR based molecular techniques. Radiological outcome was measured by scoring X-rays of the hands and feet using the standard radiographs of Larsen (Larsen score). Patients were stratified by RF status and the results were analysed using the Fisher exact test.

Results The frequency of individuals with DRB1\%04 ( $p<$ $0.00001)$ was significantly higher, DRB $1 * 05(\mathrm{p}<0.04)$ and $* 07$ $(\mathrm{p}<0.006)$ were lower in the pts in compare with the controls. HLA-DRB1*04 proved to be significantly increased in seropositive pts $(\mathrm{p}<0.038)$. $\mathrm{RF}(+)$ pts with homozygous status for HLADRB $1 * 04(p<0.04)$ had significantly higher radiological score $(p$ $<0.05)$.

Conclusion Protracted RA in the Russian population is characterised by high frequency HLA-DRB1*04 and low distribution DRB1*05 and DRB1*07. In RF(+) RA pts there was association between the presence DR4 and radiological severity.

\section{AB0035 PLATELET MICROPARTICLES IN PATIENTS WITH RHEUMATOID ARTHRITIS}

EA Knijff-Dutmer, J Koerts, EM Kalsbeek-Batenburg, MA Van de Laar. Rheumatology, Medisch Spectrum Twente, Enschede, Netherlands

\subsection{6/annrheumdis-2001.940}

Background Platelet microparticles (PMP) contain pro-coagulant as well as pro-inflammatory substances like platelet activating factor (PAF) and P-selectin. Rheumatoid arthritis (RA) is associated with increased concentrations of both PAF and P-selectin, but the link with PMP has never been investigated.

Objectives To test the hypothesis that patients with RA have elevated levels of PMPs and that these levels are associated with disease activity.

Methods This was a descriptive study in patients with RA, using neither anticoagulants nor corticosteroids. The patients were divided in two groups, according to disease activity. Criteria for active RA were: ESR at least $28 \mathrm{mmh}$ and/or CRP at least 28 $\mathrm{mg} / \mathrm{dl}$ plus more than 8 painful and more than 5 swollen joints. Criteria for RA in remission were: ESR $<28 \mathrm{mmh}$ and/or CRP $<28 \mathrm{mg} / \mathrm{dl}$ plus no painful and no swollen joints. PMPs were determined with flowcytometry (CD61/annexin-V). The values were compared with those from healthy volunteers, using nonparametric tests (Mann-Whitney).

Results Ten patients with active RA and ten patients with RA in remission were included. There were 5 men and 15 women. The mean age was 61 years, the mean disease duration was 6 years and $75 \%$ was rheumatoid factor positive (comparable in both groups). The mean number of PMPs in RA patients was $3,173 \times$ $106 / \mathrm{L}$ versus $451 \times 106 / \mathrm{L}$ in healthy volunteers $(\mathrm{p}=0.005)$. See Table 1 for further results:

\begin{tabular}{llll}
\multicolumn{4}{l}{ Abstract AB0035 Table 1 } \\
\hline & Active RA & RA in remission & Healthy \\
\hline DAS & $6.8(5.1-7.5)$ & $2.1(1.3-2.4)$ & - \\
PMPs x10 6/L & $2,104(120-22,441)$ & $504(121-5,562)$ & $118(35-1,881)$ \\
Mann-Whitney & $\mathrm{p}=0.01$ & $\mathrm{p}=0.028$ & \\
\hline
\end{tabular}

Conclusion This study confirms the hypothesis that patients with RA have higher numbers of PMPs; moreover, the presence of PMPs is associated with disease activity. This observation is most probably the missing link between the studies on the PMP content in general and the raised PAF- and P-selectin levels in sera from patients with RA. Moreover, one may speculate on the possible meaning of these elevated PMPs for the well-known high mortality due to cardiovascular disease in patients with RA. More research may clarify this.

\section{AB0036 POLYMORPHISM OF CHOLESTEROL CRYSTALAS IN SYNOVIAL FLUID}

IT Zimmermann-Górska, M Puszczewicz, G Biakowska-Puszczewicz. Rheumatology and Rehabilitation, Karol Marcinkowski University of Medical Sciences, Poznan, Poland

\subsection{6/annrheumdis-2001.94}

Background The aim of the study was to evaluate the frequency of cholesterol crystals occurrence in synovial fluid (SF) in rheumatic diseases as well as characteristics of crystals.

Objectives We have studied 1000 synovial fluid samples from 854 patients. The most numerous was group of rheumatoid arthritis (RA) patients (573 cases) and osteoarthritis (OA).

Methods Synovial fluids were taken on heparin as anticoagulant. SF sediments were examined under polarised microscope.

Results Cholesterol crystals were demonstrated in 16 samples taken from 7 patients with RA only. Most of the crystals occurred as typical large flat rectangular or romboid plates. They ranged in size from 8 to $100 \mathrm{um}$ and they showed strong birefringence with negative or positive elongation. Less often, they appeared as long rods or curved needle-shaped crystals.

Conclusion The results showed rare occurrence of cholesterol crystals in synovial fluid samples. Polymorphism of cholesterol crystals under polarised microscope may be a cause of difficulties in synovial crystals differentiation.

\section{OP0010 NEUTRALISATION OF IL-18 ACTIVITY IS A DISEASE- MODIFYING THERAPY IN THE MURINE COLLAGEN- INDUCED ARTHRITIS MODEL}

${ }^{1} \mathrm{C}$ Plater-Zyberk, ${ }^{2} \mathrm{LA}$ Joosten, ${ }^{2} \mathrm{MA}$ Helsen, ${ }^{1} \mathrm{P}$ Sattonnet-Roche, ${ }^{1} \mathrm{C}$ Siegfried, ${ }^{1} \mathrm{~S}$ Alouani, ${ }^{2}$ AJ Van de Loo, ${ }^{1} \mathrm{P}$ Graber, ${ }^{2} \mathrm{E}$ Lubberts, ${ }^{3} \mathrm{CA}$ Dinarello, ${ }^{2}$ WB Van den Berg, ${ }^{1} \mathrm{Y}$ Chvatcko. ${ }^{1}$ Experimental Biology and Pharmacology, Serono Pharmaceutical Research Institute, Geneva, Switzerland; ${ }^{2}$ Rheumatology Research Laboratory, University Medical Center StRadboud, Nijmegen, The Netherlands; ${ }^{3}$ Department of Medicine, University of Colorado Health Sciences Center, Denver, USA

\subsection{6/annrheumdis-2001.942}

Background Interleukin-18 (IL-18) is a member of the interleukin-1 cytokine family. In vivo IL-18 has been demonstrated as promoting the development of a TH1 response in synergy with IL-12. Patients with rheumatoid arthritis (RA) have in their joints significant levels of IL-18 and IL-12.

Objectives This study was aimed at studying the effect of neutralising endogenous IL-18 in vivo in collagen-induced arthritic (CIA) mice, to define the therapeutic potentials of IL-18 blockade for the treatment of patients with RA.

Methods Two distinct IL-18 neutralising strategies were used, i. e. a recombinant human IL-18 binding protein (rIL-18BP) and a polyclonal rabbit anti-mouse IL-18 IgG, to treat CIA mice in a 